\title{
LE DON AU MOYEN ÂGE
}

Pratique sociale et représentations perspectives de recherche

\author{
Eliana Magnani
}

La Découverte | « Revue du MAUSS »

2002/1 n n $^{19}$ | pages 309 à 322

ISSN 1247-4819

ISBN 2707137642

DOI 10.3917/rdm.019.0309

Article disponible en ligne à l'adresse :

https://www.cairn.info/revue-du-mauss-2002-1-page-309.htm

Distribution électronique Cairn.info pour La Découverte.

(C) La Découverte. Tous droits réservés pour tous pays.

La reproduction ou représentation de cet article, notamment par photocopie, n'est autorisée que dans les limites des conditions générales d'utilisation du site ou, le cas échéant, des conditions générales de la licence souscrite par votre établissement. Toute autre reproduction ou représentation, en tout ou partie, sous quelque forme et de quelque manière que ce soit, est interdite sauf accord préalable et écrit de l'éditeur, en dehors des cas prévus par la législation en vigueur en France. Il est précisé que son stockage dans une base de données est également interdit. 


\section{LE DON AU MOYEN ÂGE : PRATIQUE SOCIALE ET REPRÉSENTATIONS}

Perspectives de recherche ${ }^{1}$

\section{par Eliana Magnani Soares-Christen}

L'acte de donner est l'une des pratiques les plus constantes et les plus répandues du Moyen Âge. Il est aussi ce qu'il y a de plus documenté, à tel point qu'il a pu paraitre anodin. Longtemps traité dans son acception juridique ${ }^{2}$, ce n'est que récemment que le don a pris place parmi les préoccupations des médiévistes en tant que fait social.

Où que l'on regarde, de la concession de privilèges princiers au versement des redevances paysannes, de l'attribution de fiefs et bénéfices aux alliances matrimoniales, dans tous les échanges médiévaux le don est présent. Des milliers de documents écrits concernant tout l'Occident font état de donations faites aux églises et monastères. Et malgré la diversité des pratiques et l'abondance des sources, on ne s'est jamais inquiété de dépasser des modèles d'explication qui sont loin de rendre compte de la complexité du don et des échanges, ce qui nous laisse aujourd'hui devant une impasse historiographique.

Soucieux d'ouvrir l'histoire médiévale aux importants acquis de l'anthropologie, les historiens, à commencer par Georges Duby, ont utilisé des modèles d'explication dressés par les ethnologues, sans se demander s'ils étaient opérants pour la société médiévale. C'est le cas notamment de la théorie du don/contre-don de Marcel Mauss ${ }^{3}$, à partir de laquelle les médiévistes en sont venus à parler d'une économie du don, par opposition à une économie de marché, et qui, appliquée à la forme la plus répandue du don au Moyen Âge, à savoir les dons faits aux églises, a fixé la compréhension de cette pratique autour d'une image stérile : celle du don de biens matériels en contrepartie de services liturgiques. Des travaux tout à fait récents qui ont traité d'une manière ou d'une autre de la question des donations au Moyen Âge se placent toujours dans la contrainte du modèle maussien. Le plus paradoxal, c'est que certains auteurs ont abouti à des résultats qui montrent l'inadéquation du modèle à la réalité médiévale; mais ils se sont toujours efforcés de les « adapter » à ce cadre théorique contraignant.

Des recherches sur le don doivent donc commencer par résoudre ce problème historiographique et par une interrogation sur la méthode. Il ne s'agit évidemment pas de proscrire tout recours à l'anthropologie. Bien au contraire. Mais ce n'est pas en empruntant des modèles aux anthropologues que l'on fera avancer la connaissance historique. C'est leur démarche qui doit nous inspirer. Il faut ainsi écarter les anachronismes et les clivages traditionnels de l'historiographie. Il faut comprendre la société médiévale de l'intérieur, à partir de ses propres représentations. Il faut dégager les notions propres à 
rendre compte de cette société dans sa totalité. L'étude du don, puisqu'il peut être envisagé comme un départ pour appréhender les échanges en général, est sans doute l'un des moyens pour pénétrer cette société.

\section{LE CHAMP DOCUMENTAIRE ET CHRONOLOGIQUE}

Les sources nous indiquent la porte d'entrée dans l'univers des échanges, là où le don apparaît le plus dévoilé : dans les donations faites à Dieu à travers ses représentants sur terre, prêtres et moines. Les archives d'origine ecclésiastique livrent une masse de documents, les « actes de la pratique », où sont consignés les biens ou les droits abandonnés au profit d'une église, d'un monastère. Ces séries documentaires, souvent compilées dans des recueils, les cartulaires, commencent à apparaître grosso modo au $\mathrm{IX}^{\mathrm{e}}$ siècle et sont promises à un long avenir, au moins jusqu'au début du XIII ${ }^{\mathrm{e}}$ siècle. Elles couvrent, avec des variations chronologiques, l'ensemble de l'Occident latin. La longue durée de la documentation dans le temps et son large contour dans l'espace rendent compte de l'usage généralisé de l'écrit comme forme d'enregistrement du don, mais aussi de tout autre type de transaction. Elle permet de saisir à la fois la permanence et l'évolution de ces pratiques, de même que les structures communes et les variantes locales.

L'omniprésence de la donation aux églises entre le $\mathrm{IX}^{\mathrm{e}}$ et le début du $\mathrm{XIII}^{\mathrm{e}}$ siècle suppose une certaine cohérence structurelle au long de la période. En effet, les études récentes tendent à révéler les liens, voire la continuité des structures politiques et sociales entre l'époque dite « carolingienne » et l'« ordre seigneurial ». Le don, principale forme d'échange entre les familles aristocratiques et les institutions ecclésiastiques, comme l'ont montré les travaux de Barbara Rosenwein et Michel Lauwers, joue un rôle structurant. Il se constitue alors en système de fonctionnement de la société, c'est-à-dire qu'il crée et révèle des hiérarchies, fixe des identités familiales, établit des liens entre les hommes, qu'ils soient encore en vie ou déjà morts, concrétise les rapports entre l'ici-bas et l'au-delà. Au XIII siècle, l'abandon de la donation au profit de la pratique testamentaire témoigne des mutations sociales qui transforment la place, et certainement la perception, du don dans cette société.

\section{ÉGLISE ET PASTORALE : LA PRODUCTION THÉOLOGIQUE ET LES CHARTES}

Dans une société qui se définit comme communauté des chrétiens, organisée par Dieu et en relation avec lui, et dont toute hiérarchie s'établit en fonction de la capacité d'être plus au moins proche du sacré, l'Église occupe une place prépondérante; d'où le besoin de placer la théologie dans le champ d'investigation. En ce qui concerne le don, les implications 
théologiques sont nombreuses. Pour les saisir, deux démarches complémentaires se présentent. La première consiste à chercher dans la production scripturaire des auteurs chrétiens - des Pères de l'Église à Thomas d'Aquin les réflexions qui, directement ou indirectement, se réfèrent au don. C'est un vaste programme qu'il faut aborder à partir d'une piste centrale : l'offrande dans le rite eucharistique et l'aumône. La deuxième démarche consiste à déceler dans les témoins directs de la pratique du don - dans les actes les enseignements théologiques.

On sait que les dons et leur écriture ont lieu au cours de cérémonies publiques, en présence d'une assemblée plus ou moins importante, où se côtoient clercs et laïcs. Les actes de donation sont dictés, lus et entendus; certains sont même rimés, indice de leur oralité. Souvent le donateur dépose le parchemin sur l'autel consacré au saint patron de l'église. Le don est ainsi à l'origine d'un véritable rituel qui passe par la parole, parlée et écrite, et par les gestes. Il est revêtu d'un symbolisme qu'une enquête iconographique aidera à décrire et à comprendre.

Si on s'attarde sur le contenu de ces actes, notamment sur leur introduction (préambule), on observe qu'ils véhiculent une pédagogie, qu'ils sont un instrument de la pastorale ecclésiastique. On y rencontre les citations de l'Écriture qui justifient et expliquent l'action dont l'acte est la preuve ainsi qu'une série de réflexions, notamment sur la condition humaine face à Dieu, la mort, la vie sur terre et dans l'au-delà, et sur la richesse ou la justice. Ces observations sont rarement très développées, mais elles fournissent un échantillonnage des préoccupations qui circulent, ou que les clercs s'efforcent de faire circuler, dans la société. Dans tous les cas, il s'agit de discours tenus lors de la réalisation de transactions qui ont une portée concrète sur la vie matérielle de tous les jours. Le don, au-delà de tout symbolisme, est une pratique économique qui insère l'Église dans le système de production. C'est donc sa place dans le fonctionnement du monde, dans l'exploitation du travail paysan, qui est aussi en jeu et qu'il faut légitimer. Cela passe par la diffusion d'une idéologie, y compris à travers les chartes de donation. L'intérêt de l'établissement d'une typologie des thèmes traités dans les préambules des actes est de dégager les différentes formulations idéelles suscitées par le don, et de comprendre comment elles ordonnent la société.

\section{LE DON PRO ANIMA : OFFRANDE ET AUMONNE}

Ainsi, à partir de l'analyse des préambules des chartes de donation provençales et clunisiennes des $\mathrm{XI}^{\mathrm{e}}$ et $\mathrm{XII}^{\mathrm{e}}$ siècles ${ }^{5}$, épaulée par la réflexion issue de quelques travaux récents, il est possible de dépasser les explications inspirées de la théorie du don/contre-don (don de biens matériels en contrepartie de services liturgiques) et de montrer la multiplicité des implications d'une pratique bien plus complexe. En fait, les donations pro anima impliquent 
dans l'échange d'autres acteurs que le donateur et les religieux, faisant intervenir également les pauvres et les morts (les « ancêtres», les saints). Et surtout, les documents révèlent le phénomène de la circulation des biens et des personnes entre l'ici-bas et l'au-delà, circulation rendue possible grâce à la transformation des biens terrestres en biens célestes, et du pécheur en homme nouveau. Les donations sont ainsi inscrites dans un double registre, fonctionnant à la fois comme aumône et comme offrande eucharistique.

Des temporalia vers les cælestia. La circulation des biens entre l'ici-bas et l'au-delà.

Le concept du don pro anima parait assez simple et clair : il s'agit d'un acte rédempteur. Son but est de mettre en route les mécanismes et les acteurs qui garantissent au donateur sa place dans le Ciel. L'Écriture fournit les fondements d'une telle démarche. Tout d'abord le précepte biblique de l'aumône. En fait, parmi les passages scripturaires les plus cités, le Siracide 3, 30, rappelle la faculté qu'a l'aumône d'effacer les péchés, comme l'eau éteint le feu ${ }^{6}$, et dans Luc 11,41 , on promet tout à celui qui fait l'aumône ${ }^{7}$. L'aumône est, dans la tradition chrétienne, le don fait aux pauvres ${ }^{8}$. C'est le devoir de charité (caritas) de tout chrétien envers son semblable, la charité étant comprise comme l'amour chrétien - celui de Dieu envers les hommes et celui des hommes envers Dieu et envers les autres hommes ${ }^{9}$. La distribution de l'aumône est l'une des responsabilités des églises au Moyen Âge, dont une partie du patrimoine est considérée comme propriété des pauvres. Le don aux monastères vise, par l'intermédiaire des moines, à accomplir l'obligation d'assistance aux nécessiteux, de pratiquer la caritas, de reproduire l'amour de Dieu qui « a tant aimé le monde qu'il lui a donné son Fils unique » $(\mathrm{Jn} 3,13)$. La charité est la condition de la valeur spirituelle de l'aumône.

Le pauvre - on y reviendra - est un acteur fondamental de l'échange car il est le véhicule du salut du riche. Effectivement, dans un passage de la Vie de saint Éloi, écrite dans le premier quart du VIII ${ }^{\mathrm{e}}$ siècle, on souligne que « Dieu aurait pu faire tous les hommes riches, mais il voulut qu'il y ait des pauvres en ce monde, afin que les riches aient une occasion de racheter leurs péchés ${ }^{10} \gg$. Par la volonté du Créateur de sauver les riches, la société est fondée sur l'inégalité. Ainsi, les biens dont dispose le riche sont, en fin de compte, le moyen de son salut ${ }^{11}$. La même idée apparaît dans l'acte de fondation de Cluny qui commence par le rappel suivant : «Si la Providence de Dieu veut qu'il y ait des hommes riches, c'est afin qu'en faisant un bon usage des biens qu'ils possèdent de façon transitoire, ils méritent des récompenses qui dureront toujours ${ }^{12}$. »

La donatio pro anima s'articule, en effet, autour de l'idée que Dieu a donné aux hommes la possibilité d'obtenir des biens célestes grâce aux biens terrestres, de construire des choses éternelles avec des biens passagers ${ }^{13}$. Des temporalia, à condition qu'on en fasse un bon usage, peuvent se 
transformer en caelestia $^{14}$. En ce sens, un acte de Lérins datant de 1033 fait remarquer que parmi les miracles de l'œuvre du « grand arbitre » et les merveilles qu'il a données aux mortels figure le fait que ceux-ci puissent mériter les éternelles et perpétuelles récompenses grâce aux biens matériels et fragiles ${ }^{15}$. L'affirmation se trouve confortée par l'Écriture qui enseigne qu' « on récolte ce qu'on sème » $(\operatorname{Pr} 22,8 ; \mathrm{Si} 7,3 ; \mathrm{Jb} 4,8 ; \mathrm{Os} 8,7 ; \mathrm{Ga} 6$, $7 ; 2$ Cor 9,6 ) et que « la justice de celui qui a distribué et donné aux pauvres de ses biens demeure à jamais »(Ps 112, 9; 2 Co 9, 9). Donc on est en droit d'espérer retrouver dans l'au-delà ce qu'on donne ici-bas.

La circulation des biens entre ces deux sphères est envisagée de manière tout à fait concrète dans un passage de la chronique galicienne d'Iria ( $\mathrm{CrO}$ nicón Iriense) rédigée dans le dernier tiers du $\mathrm{XI}^{\mathrm{e}}$ siècle, probablement dans un milieu monastique ${ }^{16}$. Il s' agit des deux visions de la reine Godo, épouse du roi Sancho Ordóñez de Galice ${ }^{17}$ (†929). Le roi, grand bienfaiteur des églises, meurt, victime d'empoisonnement, dans les environs du monastère de Castrelo del Miño, où sa femme Godo l'enterre et où elle prend le voile. Un samedi, pendant qu'elle priait devant l'autel, son mari apparaît enchaîné, tenu par deux démons, et lui dit qu'elle est en train de l'aider et qu'elle doit persévérer ( «benefacis » et «persevera »). Elle continue donc à jeûner, à verser des larmes et à faire l'aumône (in ieiunio et fletu et in elemosinis largiendis). Après quarante jours, un autre samedi, pendant qu'elle priait à l'autel, Sancho lui apparaît une nouvelle fois habillé en blanc et portant la pelisse que Godo avait donnée à un prêtre comme suffrage pour l'âme de son mari. Celui-ci manifeste sa satisfaction d'avoir été libéré de l'emprise du démon et raconte plusieurs choses sur le paradis et l'enfer. Alors la reine essaie, sans succès, de l'embrasser, mais un bout de la fourrure lui reste entre les mains. Elle amène le morceau de peau au monastère de Saint-Étienne de Ribas de Silo où on se rend compte que dans la pelisse que Godo avait donnée au prêtre manquait en effet le morceau qu'elle apportait.

Outre la valeur de la prière et de l'aumône pour le salut des défunts, le récit des visions de la reine Godo montre que les biens donnés ont une double existence, ici-bas et dans l'au-delà, et qu'ils transitent entre un monde et l'autre. Un passage de la Vie de l'empereur Henri II, écrite vers le milieu $\mathrm{du} \mathrm{XII}^{\mathrm{e}}$ siècle, dévoile une idée similaire ${ }^{18}$. Juste après la mort de l'empereur, le démon a révélé à un ermite que quand les mérites d'Henri ont été pesés, ses péchés avaient fait pencher la balance du côté de l'enfer. Mais, soudain, quelqu'un est apparu et a jeté un calice en or du côté de l'ange, changeant si miraculeusement le fléau de la balance que celle-ci est tombée par terre et que le calice s'est fendu. Il s' agissait du calice qu'Henri avait donné à Saint-Laurent de Mersebourg et qui plus tard a été trouvé ébréché dans l'église où il était gardé sous clé. Grâce au don de cet objet eucharistique, l'âme de l'empereur a pu aller au paradis. Le calice en or offert par Henri II était passé dans l'au-delà où il agit littéralement par son «poids » en faveur de l'âme de l'empereur pour ensuite retrouver sa place dans l'église 
cathédrale, tout en portant les traces des aléas subis lors de son périple. Donc les biens donnés ont une double existence, ici-bas et dans l'au-delà, et ils transitent entre un monde et l'autre.

Avec ce calice, Henri avait ajouté une pièce au trésor de Saint-Laurent; mais surtout, pour reprendre les expressions scripturaires utilisées dans les actes de donation, il s'était fait des « amis » et constitué « un trésor dans le Ciel ». Celui qui apparaît et jette le calice dans la balance intervient définitivement dans le destin de l'âme de l'empereur. Cet « ami », personnage non identifié - peut-être un clerc ou saint Laurent lui-même? - est là pour faire valoir dans le Ciel le « trésor » constitué par le donateur. Un trésor où, à côté des objets précieux meubles, figurent aussi les terres et droits concédés aux églises. Dans la liste des biens donnés par Henri II à l'évêché de Mersebourg dressée vers 1136 dans la «Chronique des évêques de Mersebourg », on énumère trois calices dont l'un est décrit comme étant soigneusement manufacturé avec toutes sortes de gemmes et comme étant « le prix de la rédemption » de l'âme de l'empereur. Plus loin dans la liste, la même expression sert à désigner six domaines qu'Henri avait également offerts $^{19}$. Dans cette première moitié du XII ${ }^{\mathrm{e}}$ siècle, pour les ecclésiastiques de Mersebourg, il était important de souligner la valeur rédemptrice des possessions thésaurisées à la fois ici-bas par l'Église et, par son intermédiaire, dans l'au-delà par le donateur.

\section{Conversion du pécheur, transformation des biens et « commutation » eucharistique}

La donation pro anima permet donc au donateur de constituer un « trésor dans le $\mathrm{Ciel}^{20}$ ». Mais les biens terrestres doivent être transformés en récompense céleste. Et cette transformation ne se limite pas pour autant à celle des choses données, car elle implique la conversion du donateur de pécheur en homme nouveau. Augustin, en rattachant l'efficacité rédemptrice de l'aumône à la véritable conversion du cœur et au changement dans la conduite du donateur, ouvre la voie à un « courant spiritualiste de l'aumône » qui met en cause ceux qui absolvent tout pécheur en raison des aumônes effectuées par lui, sans évaluer ses fautes ou son genre de vie présent $^{21}$. Ce débat est toujours d'actualité au XI ${ }^{\mathrm{e}}$ siècle où la « qualité » du don, pur ou impur en fonction des actes du donateur, semble être un nouveau sujet d'inquiétudes.

La conversion du donateur est au centre des préoccupations de l'acte de donation passé en 1014 par le vicomte Guillaume II de Marseille en faveur de l'abbaye de Saint-Victor. Le rédacteur rappelle, à l'appui d'un passage extrait du livre du prophète Ézéchiel $(23,11)$, que Dieu veut que le pécheur puisse atteindre la vie éternelle. Mais pour que le Seigneur exerce sa miséricorde, pour qu'en définitive il accorde son pardon, le pécheur doit changer de conduite : il faut «qu'il se convertisse » (ut convertatur). La transformation de l'homme est donc au départ de son salut. Les moyens de 
cette conversion, comme le suggère la suite du même préambule, se trouvent dans la donation de biens terrestres avec lesquels le vicomte espère pouvoir obtenir les biens célestes (ut pro terrenis celestia adquirere valeam). La transformation de l'homme passe par celle de ses biens, des biens qui, comme le souligne Guillaume, ont été reçus du Seigneur lui-même (de ea que illi mihi contulit ${ }^{22}$ ). Six ans auparavant, lors d'une autre donation de Guillaume et de ses frères, on rappelle que donner ce qu'on reçoit permet de réaliser l'heureuse commutation (felice commutatione) de ce qu'on ne peut que temporairement posséder, et avoir la jouissance en récompense pour l'éternité, car, comme il est dit dans le livre de l'Apocalypse, « les œuvres suivent ceux qui les ont accomplies ${ }^{23} »($ Ap 14, 13).

À l'instar des actes de Cluny relevés par Dominique Iogna-Prat, ces exemples révèlent, dans les chartes, l'emploi des vocables qui associent la donation à la transformation des biens : donare atque transfundere, tradere atque transfundere, transmutatio, commutatio, inmutatio ${ }^{24}$. Il s'agit, comme dans cet acte clunisien, de transformer les personnes et les biens en «quelque chose de mieux » (in melius transmutando ${ }^{25}$ ). Le vocabulaire de la transformation place la donation dans un contexte eucharistique, à une époque où les problèmes doctrinaux autour de l'eucharistie, les débats entre symbolistes et réalistes, ne sont pas encore réglés ${ }^{26}$. Dominique Iogna-Prat a montré notamment que les vocables employés dans l'œuvre polémique de Pierre le Vénérable dans sa réflexion eucharistique renvoient aux notions de mutatio, commutatio, transmutatio, ce qui permet de placer «le "sacrement de l'autel" dans l'ample catégorie des "miracles" et "mutations" s'accomplissant au quotidien ${ }^{27} »$. Cela revient par conséquent à concevoir que la transformation des biens et des personnes opérée grâce à la pratique du don est de nature similaire à celle de la «commutation » eucharistique.

Le cadre solennel où se réalisent les donations tend en effet à reproduire l'eucharistie, ou s'en inspire. Le dépôt d'une charte de donation, ou de restitution, sur l'autel, endroit voué au sacrifice et à la transformation, parfois à l'occasion d'une cérémonie publique, est une pratique attestée par les formulaires carolingiens et présente dans nos documents ${ }^{28}$. Dès le $\mathrm{VI}^{\mathrm{e}}$ siècle, des objets à connotation eucharistique - calice, patène, livres liturgiques - sont directement attachés aux donations ${ }^{29}$. Les donations et redevances en vin et en blé attribuées aux moines sont également en rapport, direct ou indirect, avec l'offrande eucharistique ${ }^{30}$.

L'intercession, pour l'âme du donateur lors de la célébration eucharistique, est au centre de la donation faite à Saint-Victor de Marseille en 1054 par un certain Guérin, avec son épouse et ses enfants. Guérin donne des vignes et reçoit en contrepartie de son don un splendide missel, d'une valeur de cent sous, qu'il offre ensuite à Saint-Victor et aux églises du Saint-Sépulcre et de Saint-Mitre, pour pouvoir bénéficier des prières des moines et de celles qui seraient dites avec ce missel ${ }^{31}$. Les biens de Guérin avaient ainsi servi à l'acquisition d'un missel - livre liturgique qui n' apparaît en Occident qu' au $\mathrm{XI}^{\mathrm{e}}$ siècle et dont on a ici un témoignage précoce - 
grâce auquel les religieux allaient pouvoir s'occuper de son âme pendant la messe.

Très significative également est la pratique qui consiste à écrire son nom directement sur les autels. Thietmar de Mersebourg raconte qu'un abbé de Berge cultivait cette habitude ${ }^{32}$. Une table d'autel retrouvée en 1976 lors de fouilles de Reichenau-Niederzell contient 203 noms gravés et 131 inscrits à l'encre. Plusieurs noms ont pu être mis en relation avec certaines inscriptions du liber memorialis, et datées ainsi des années $970-972^{33} . \mathrm{La}$ table d'autel du ve siècle de l'église Saint-Pierre d'Auriol en Provence est recouverte de deux inscriptions plus récentes où figurent une dizaine de noms $\mathrm{s}^{34}$. Inscrivant son nom sur l'autel, on s'associait personnellement à la transformation réitérée à chaque jour par la célébration du sacrifice.

La connotation eucharistique et solennelle du don pro anima révèle que, d'une manière générale, la donation est bien plus qu'une aumône : elle est également une offrande destinée à la célébration du sacrifice. Le don permet ainsi au donateur laïc d'intégrer la communauté rituelle du monastère et de prendre place dans les échanges avec Dieu. Car l'homme médiéval est conscient de la dette envers Celui qui a donné à l'homme toutes les richesses terrestres ${ }^{35}$, dont on est débiteur et qu'il faut rétribuer ${ }^{36}$. Conscient aussi que tout ce que l'homme peut offrir est bien peu face à ce que le Créateur lui a accordé ${ }^{37}$. Comme l'a fait remarquer récemment Maurice Godelier ${ }^{38}$, l'échange avec la divinité est fondamentalement inégal : il n'y a pas de contre-don équivalent aux dons faits par Dieu.

\section{Dieu et saints, débiteurs du donateur}

Il n'en reste pas moins que la dette a également été envisagée en sens inverse. Dans certains actes clunisiens - et cela jusqu'au deuxième tiers du $\mathrm{XI}^{\mathrm{e}}$ siècle, la donation obligerait les saints patrons des monastères, devenus débiteurs du donateur, à témoigner de ses bonnes œuvres lors du jugement dernier ${ }^{39}$. Pierre Damien (†1072) soutient l'idée que l'aumône parvient à faire de Dieu le débiteur de l'homme charitable ${ }^{40}$. Dans une lettre de 1064 à l'évêque d'Urbino, il raconte qu'un certain marquis Bernard, originaire de Germanie, possédait des nombreux biens et douze fils. Songeant aux rivalités que, après sa mort, le partage de ses possessions engendrerait entre les nombreux cohéritiers, Bernard décide de donner tous ses biens à Dieu. Bernard s'adresse alors au Seigneur, annonçant qu'il L'avait choisi comme son seul héritier et qu'il recommandait ses fils, ainsi dépossédés, à Sa Bienveillance. Ensuite Bernard attache un gant à une flèche et la lance loin vers le Ciel. La flèche retombe aussitôt, mais sans le gant, indice que Dieu avait accepté le don. Bernard transfère alors ses biens à une église, et peu de temps après, une multitude de biens commencent à affluer vers ses enfants, qui constituent une fortune plus grande que celle de leur père. En fait, le gant - emblème de l'investiture - reçu au Ciel de la main du père s'est répandu en bénédictions sur ses fils. Selon Pierre Damien, à l'instar 
des mots de l'ange à Corneille dans les Actes des Apôtres (10,31), ce qui est donné à Dieu sur terre, est reçu au Ciel. Et on peut alors attendre du Ciel un prix qui dépasse ce qui a été donné. Si l'homme pense à faire des dons, il peut faire de Dieu son débiteur et Lui demander en échange des compensations à cette dette ${ }^{41}$.

Le récit de Pierre Damien est un exemple supplémentaire des échanges concrets entre terre et Ciel. Ici le marquis négocie directement avec Dieu selon des modalités qui rappellent les échanges typiques de la vassalité. Dieu, ayant conservé le gant que Bernard envoya dans le Ciel comme symbole de l'investiture de ses biens, accepte de devenir l'obligé, l'avoué de Bernard. En tant que son débiteur, Dieu veille à rétribuer le don par des bénédictions sur ses fils. Et il ne s'agit pas seulement de les récompenser par des biens spirituels, mais de remplacer - en mieux - l'héritage terrestre duquel ils ont été privés à cause du don fait par leur père. Pierre Damien, par cet exemple, adapte au contexte féodal d'anciennes conceptions patristiques sur l'aumône. Les Écritures rappellent à plusieurs reprises les bénédictions avec lesquelles Dieu récompense l'homme qui vient au secours du pauvre et des faibles (Dt 14, 29; Sr 7, 32; 12, 2; Ps 41, 2-3; Pr 3, 27-33; 22, 9; II Cor 9, 6-15; etc.). Dans le livre des Proverbes apparaitt l'idée que ces récompenses se traduisent aussi par la multiplication des biens terrestres (Pr 3, 9-10) et que l'aumône faite au pauvre est un prêt fait au Seigneur ( $\operatorname{Pr}$ 19, 17). C'est d'ailleurs ce dernier passage que Pierre Damien cite dans une lettre de 1070 où il reprend la formule du Dieu débiteur ${ }^{42}$. En fait, Pierre Damien assure le relais des Pères grecs et latins - Jean Chrysostome, Cyprien ou Ambroise - qui soutiennent que, par l'aumône, l'homme prête à Dieu, faisant de Lui son débiteur ${ }^{43}$. N'empêche que ce prêt est une sorte de placement et que Dieu rend, ici-bas ou dans l'au-delà, toujours plus que ce qui Lui a été donné. Même si le don peut être envisagé comme un «heureux commerce », passible de contraindre Dieu à distribuer des bénédictions, l'échange reste inégal.

Il semblerait, mais il faudrait approfondir les recherches, que le thème de la dette des saints, de Dieu et de leurs serviteurs demeure assez marginal. Il n'apparaît plus dans les actes clunisiens dès la fin du XI $\mathrm{XI}^{\mathrm{e}}$ siècle et est absent des chartes provençales. En tout cas, ce n'est jamais un argument isolé puisque la valeur spirituelle et rédemptrice de l'aumône est, depuis Augustin, systématiquement rappelée, comme le fait d'ailleurs Pierre Damien dans sa lettre de 1070 .

\section{La médiation nécessaire}

Même si le marquis Bernard - selon la lettre de Pierre Damien - a pu « négocier » directement avec Dieu la donation de ses biens, pour la rendre effective, il a dû les transférer à une église. En effet, le don s'adresse concrètement aux moines-prêtres - serviteurs de Dieu - qui contrôlent les moyens pour établir des rapports avec l'au-delà. Ce sont eux les sacrificateurs 
qui rendent possible la conversion de l'homme et de ses biens, comme celle du pain et du vin en corps et sang du Christ, à travers le service de l'autel. Dans les actes, ils sont appelés à intercéder pour le pardon des péchés des donateurs ${ }^{44}$, ils sont les « amis », de même que les pauvres et les saints qui, selon l'Évangile de Luc, « lors que les richesses viendront à manquer, recevront le riche dans les tabernacles éternels ${ }^{45} »(\operatorname{Lc} 16,9)$. Des amis que l'on peut se faire grâce « aux richesses injustes ». La donation, semblable à l'offrande lors de la messe, sert ainsi à créer le lien; elle établit l' " amitié » entre le donateur et ceux qui sont chargés de reproduire les mécanismes de son salut. Nous venons de voir que ce lien a pu être formulé en termes de dette, dans un contexte qui évoque les rapports vassaliques. Mais qu'il s'agisse de se faire des « amis » ou des « obligés/avoués », la donation engage les représentants de Dieu à opérer le passage de l'homme, à travers ses biens, d'ici-bas vers l'au-delà, afin qu'il puisse être accueilli, selon l'Évangile de Matthieu, dans le « royaume qui lui a été préparé dès la fondation du monde ${ }^{46} \gg($ Mt 25, 34). À la fois aumône et offrande, le don transformé convertit le pécheur en homme nouveau; c'est un garant de la vie après la mort.

Mais d'ordinaire, les dons effectués du vivant du donateur sont rarement suffisants pour conduire l'âme au paradis; d'où l'importance des suffrages et des prières des vivants pour les morts et de l'instauration d'aumônes destinées aux pauvres et qui continuent à être distribuées après la mort du donateur, associées souvent à sa commémoraison ${ }^{47}$. On connaît le nombre impressionnant de prébendes affectées aux nécessiteux à Cluny et la fonction d'assistance exercée par les monastères ${ }^{48}$. Subvenir à l'entretien d'un pauvre équivaut à renouveler dans le temps les effets de ses bonnes œuvres, de continuer à agir ici-bas - contournant d'une certaine façon la certitude qu'après la mort, il n'est plus possible de faire le bien ${ }^{49}$. Comme les suffrages, le secours offert au pauvre se transforme en bienfait pour le mort. Mais ici comme ailleurs, la médiation des moines - distributeurs d'aumône - est indispensable, des moines qui sont eux-mêmes, par analogie, des pauvres et qui comme eux accueillent le mort dans les demeures célestes. Dans les échanges avec l'au-delà, le pauvre se démultiplie : on lui prête à la fois l'habit du mort et celui du moine ${ }^{50}$.

Le don est à la base d'un système d'alliance qui implique le transfert de droits et de propriétés vers les communautés religieuses et l'imbrication étroite des possessions laïques et monastiques. La thématique de la transformation, liée au sacrifice de l'eucharistie, apporte une justification fondamentale à la possession des biens par les monastères, surtout lorsque l'Église essaie, à partir du milieu du $\mathrm{XI}^{\mathrm{e}}$ siècle, de rompre leur intrication en distinguant les limites entre le domaine laïc et le domaine ecclésiastique. Les biens donnés aux monastères, puisqu'ils sont convertis en caelestia, deviennent des spiritualia, des biens rattachés à l'autel, à l'instar des castella et des sanctuaires appartenant au Mont-Cassin dont les noms ont été gravés sur les portes de bronze de l'église abbatiale ${ }^{51}$. Les 
biens appartenant aux monastères sont, en quelque sorte, déjà retournés vers Dieu. Et ce passage, cette conversion, change irréversiblement le statut des biens donnés. En insistant sur leur caractère « religieux », on soustrait théoriquement les biens ecclésiastiques du circuit des échanges, on les met à part. Cela signifie que les monastères et leurs possessions constituent ici-bas un espace articulé mais séparé, préfiguration de l'audelà. Toute confusion entre les biens laïcs et ecclésiastiques devient ainsi absurde, renforçant la prépondérance du clergé dans une hiérarchie fondée sur le sacré.

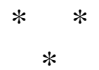

Parce que le don pro anima est représenté comme un échange avec Dieu, ses enjeux dépassent le cadre de la réciprocité inhérente à la notion de don et contre-don. La donation aux communautés religieuses est une façon d'établir la « circularité » des échanges, bien qu'inégaux, entre l'ici-bas et l'audelà, en vue de la vie éternelle. Dans un cadre chronologique qui a pour terme le jugement dernier, les échanges impliquent la transformation du bien donné afin qu'il puisse être retrouvé, en mieux, dans le royaume céleste, pour qu'il puisse constituer un « trésor dans le Ciel». La conversion des biens, à l'instar de celle du pain et du vin en corps et sang du Christ, passe par la célébration du sacrifice eucharistique et ne peut être accomplie sans la médiation des moines-prêtres, ces « amis » qui, assimilés aux pauvres, accueillent l'âme du donateur dans les «tabernacles éternels ». À la fois aumône et offrande, le don « pour le salut de l'âme » a une fonction rédemptrice et rituelle : il permet d'effacer les péchés en reproduisant la caritas divine et de sacrifier au nom du Seigneur. En se posant comme intermédiaire nécessaire dans l'exercice de la charité - dans le secours des pauvres et dans la célébration de l'eucharistie, le clergé contrôle les « commutations » propres à préparer le salut des laïcs. Le don, instaurateur de l'alliance entre laïcs et ecclésiastiques qui permet de justifier et de reproduire l'ordre seigneurial, s'organise dans un système qui, par ses représentations, tend à reproduire également les différences fondamentales qui les distinguent et qui autorisent les serviteurs de Dieu à revendiquer leur place à la tête de l'organisme chrétien.

L'importance de la donation dans l'économie de l'au-delà est certainement l'une des raisons de la diffusion de la pratique du don et de sa transformation en modèle de transaction. En effet, beaucoup d'actes de donation semblent recéler d'autres types d'opérations. L'association assez fréquente du verbe «donner » à un ou plusieurs autres verbes pour décrire l'action du sujet qui donne (ego dono, cedo, reddo, transfundo, vendo, etc.) atteste la volonté probable de ramener n'importe quelle transaction au don. Par ailleurs, 
on rencontre souvent la donation combinée avec une ou plusieurs autres sortes de transaction dans des arrangements très compliqués ${ }^{52}$. On se rend compte, du moins en ce qui concerne les églises, que l'on conçoit difficilement l'échange en dehors de la donation. Tout échange devient un prétexte pour le don. Mais on n'a pas encore déterminé comment et pourquoi le don a pénétré et modifié la conception de pratiques comme la vente, l'échange ou la restitution. Il faudra comprendre comment et dans quel sens ces opérations ont été redéfinies, en établissant une typologie du don et de ses dérivés. L'enquête, en effet, ne fait que commencer...

\section{NOTES}

1. Version remaniée de l'essai publié dans CEM (bulletin du Centre d'études médiévales d'Auxerre), $n^{\circ}$ 4, 2000, p. 62-74.

2. Par exemple, G. ChEvrier, «Évolution de la notion de donation dans les chartes de Cluny du IXe à la fin du XII e siècle », À Cluny. Congrès scientifique, Dijon, 1950, p. 203-209.

3. M. MAuss, «Essai sur le don : forme et raison de l'échange dans les sociétés archaïques », Sociologie et Anthropologie, Paris, 1950, p. 145-279; C. LÉVI-STRAUSS, «Introduction à l'œuvre de Marcel Mauss », Sociologie et Anthropologie, Paris, 1950, p. IX-LII; G. DUBY, Guerriers et paysans. VII ${ }^{e}$-XII ${ }^{e}$ siècle, premier essor de l'économie européenne, Paris, 1973, p. 60 sq.; Ph. Jobert, La Notion de donation. Convergences 630-750, publications de l'université de Dijon, 1977. Une bonne synthèse de l'utilisation du modèle du don/contredon par les médiévistes est donnée par B. Rosenwein, To be the Neighbor of Saint Peter. The Social Meaning of Cluny's Property, 909-1049, Ithaca/Londres, 1989, p. 125-143. Depuis, d'autres historiens ont abordé le problème dont M. LaUwers, La Mémoire des ancêtres, le souvenir des morts. Morts, rites et société au Moyen Âge (diocèse de Liège, XI ${ }^{e}-$ XIII ${ }^{e}$ siècles), Paris, 1997, p. 172 sq., et D. IoGNA-PRAT, Ordonner et Exclure. Cluny et la société chrétienne face à l'hérésie, le judaïsme et l'islam (1000-1150), Paris, 1998, p. 211-217.

4. Les considérations qui suivent sont extraites d'un article à paraître (« Transforming things and persons. The gift pro anima in the $\mathrm{XI}^{\text {th }}$ and $\mathrm{XII}^{\text {th }}$ century », Negotiating the Gift, G. AlGAZI, V. GRoEbNer, B. JusSEN). Les notes ont été allégées.

5. Le corpus étudié se constitue des actes des cartulaires provençaux des abbayes de SaintVictor de Marseille (compilés dans les années 1080; 900 actes), de Lérins (transcrits dans les années 1160; 290 actes) et des actes originaux (500) de l'abbaye de Cluny en Bourgogne pour un total d'environ 1700 actes, datant de la deuxième moitié du $\mathrm{X}^{\mathrm{e}}$ jusqu'au début du $\mathrm{XII}^{\mathrm{e}}$ siècle. Parmi ces nombreux actes, très peu contiennent des préambules développés, ce qui réduit considérablement le nombre des pièces utilisées : environ une soixantaine. L'enquête sur les actes clunisiens est celle de D. IOGNA-PRAT, Ordonner et Exclure, p. 211-217. Les autres références sont les suivantes : Cartulaire de l'abbaye de Saint-Victor de Marseille, éd. B. Guérard, Paris, 1857, 2 vol. (Collection des Cartulaires de France, t. VIII) [désormais nommé CSV]; Cartulaire de l'abbaye de Lérins, éd. H. Moris, E. Blanc, Paris, 1883-1905, 2 vol. [désormais CL]; Recueil des chartes de l'abbaye de Cluny, éd. A. Bernard, A. Bruel, Paris, 1871-1903, 6 vol. (Collection de documents inédits sur l'Histoire de France) [désormais CLU].

6. Si 3, 30, dans CSV 225 (1024), 165 (1028), 56, 57 (1035), CL 230 (1022), 144 (1061), CLU 2731 (v. 1020), 2815 (1029), 2997 (1049-1109), 3404 (1065) etc. Et dans le même sens : Dn 4, 24, dans CL 144 (1061), CLU 3315 (v. 1050), 3676 (1094).

7. Lc 11, 41, dans CSV 101 (1033), 123 (1035), CL 272 (1064), 244 (1080-97), 208 (111015), CLU 1715 (985), 1784 (988), 2731 (v. 1020), 2815 (1029), 2997 (1049-1109), 3350 (v. 1055), 3410 (1066), etc. 
8. Mt 19, 21 dans CL t. II n ${ }^{\circ} 28$ (1022); 2 Co 9, 9, dans CL 154 bis (1033); CSV 158 (v. 1060).

9. Sur la caritas en tant que paradigme du lien social dans la société médiévale, voir A. GUERREAUJALABERT, «Caritas y don en la sociedad medieval occidental », Hispania, vol. 60-1, $n^{\circ} 204$, p. 27-62, et « Spiritus et caritas. Le baptême dans la société médiévale », dans $L a$ parenté spirituelle, F. HÉRITIER-Augé, E. COPET-RougIER (sous la dir. de), Paris/Bâle, 1995, p. 133-203. Bien que concernant l'époque moderne, voir aussi B. Clavero, La Grâce du don. Anthropologie catholique de l'économie moderne, Paris, 1996, p. 163-175.

10. Vita sancti Eligii episcopi noviomensis, Migne, Patrologia Latina, t. 87, c. 533.

11. Pr 13, 8 dans CL 232 (milieu XI s.), 208 (1110-1115), CLU 1715 (985), 1784 (988), 3350 (v. 1055), 3506 (1077).

12. CLU 112 (910).

13. CLU 1189 (965/966), 1190 et 1191 (965/966), 2731 (v. 1020), 3377 (1061-73), 3506 (1077).

14. CSV 110 (1014).

15. CL 154 bis (1033).

16. M. C. Díaz y Díaz, Visiones del Más Allá en Galicia durante la Alta Edad Media, SaintJean de Compostelle, 1985 (Bibliofilos gallegos. Biblioteca de Galicia, XXIV), p. 66-69.

17. M. C. Díaz y Díaz, Visiones del Más Allá, p. 74-75. Ce récit mélange des faits de la vie du Sancho Ordóñez de Galice et du roi Sancho I de Léon (voir p. 65-66).

18. Adalberti Vita Heinrici II imperatoris, éd. G. WAITZ, Monumenta Germaniae Historica, Scriptores, 4, Hanovre, 1841, p. 792-820 (ici p. 810-811). Voir Ph. Buc, « Conversion of objects », Viator, 1997, p. 99-143, qui commente ce passage (p. 138-139).

19. Chronica episcoporum Merseburgensium 4, éd. R. Wilmans, Monumenta Germaniae Historica, Scriptores, 10, p. 176-177.

20. Lc 12, 33 et Mt 6, 20, dans CSV 238 (1056), CLU 1644 (983/984), 1784 (988), 2731 (v. 1020), 2815 (1029), 3350 (v. 1055), 3377 (1061-73), 3404 (1065), Mt 19, 21, dans CL t. II ${ }^{\circ} 28$ (1022), ou encore Mt 19, 29, dans CL 231 (ap. 1024).

21. Ph. Jobert, La Notion de donation..., p. 172 sq. L'étude sémantique effectuée par Bernhard Jussen dans les traités théologiques et les sermons antiques et médiévaux montre que le terme structurant du don (munus) est le cœur (cor), voire l'âme (anima, mens) (Negotiating the Gift, op. cit).

22. CSV 110 (1014)

23. CSV 113 (1008).

24. CLU 821, 1639 (983), 1668 (984), 3301 (1049-1109), 3377 (1061-73), 3585 (1081), 3676 (1094).

25. CLU 3301 (1049-1109).

26. H. DE Lubac, Corpus Mysticum. L'Eucharistie et l'Église au Moyen Âge. Étude historique, Paris, 1944.

27. D. IOGNA-PRAT, Ordonner..., p. 212-213.

28. Monumenta Germaniae Historica, Leges nationum Germanicarum, V, 1 (leges Alemannorum), p. 63-64. Voir CL 77 (1070), CSV 38 (1079), CL 215 (1125-29), 137 (1131). D'autres exemples dans F. Bougard, «Trésors et mobilia italiens du haut Moyen Âge », Les Trésors de sanctuaires, de l'Antiquité à l'époque romane, éd. J.-P. CAILLET et P. BAZIN, université de Paris-X, 1996 (Centre de recherche sur l'Antiquité tardive et le haut Moyen Âge, Cahier VII), p. 161-197 (ici p. 167-168, note 44-47).

29. Quelques exemples dans : Testamentum s. Remigii, éd. B. Krusch, Monumenta Germaniae Historica, Scriptores rerum merovingicarum, 3, Hannover, 1896, p. 337 (IX s.); CLU 3445 (v. 1090); The Ecclesiastical History of Orderic Vitalis, éd. et trad. anglaise M. CHIBNALL, Oxford, 1970, vol. III, livre V, ch. 19, p. 184 (v. 1105).

30. CLU 269 (926).

31. CSV 413 (26 mars 1054).

32. Thietmar de Mersebourg, Chronicon VIII, 11, éd. R. Holtzmann, Monumenta Germaniae Historica, Scriptores rerum germanicarum, n.s. 9, Berlin, 1935 (rééd. 1955), p. 506. 
33. Die Altarplatte von Reichenau-Niederzell, éd. D. GeUENICH, R. NEUMÜLLERS-KLAUSER, K. SchміD, Monumenta Germaniae Historica, Libri memoriales et necrologia, n. s. 1, Supplementum, 1983.

34. H. LECLERCQ, «Auriol », Dictionnaire d'archéologie chrétienne et de liturgie, I, 2, 1907, c. 3151-55. Voir aussi l'exemple de Minerve, dans l'Hérault («Graffite», ibid., VI, 2, 1925, c. 1501-1512).

35. CLU 141 ( $\mathrm{X}^{\mathrm{e}} \mathrm{s}$ ), CSV 57, 123 (1035), 238 (1056), CL 154 bis (1033), 208 (1110-1115), 238 (1094).

36. CLU 112 (910), CSV 48 (1030), 189 (v. 1030), 284 (1076).

37. CLU 3347 (v. 1054), 3557 (v. 1080).

38. M. Godelier, L'Énigme du don, Paris, 1996, p. 44-47, 249-275.

39. CLU 1999 (993-1048), 1715 (985), 2831 (v. 1021), 3350 (v. 1055), 3404 (août 1065).

40. Ph. Jobert, La Notion de donation..., p. 199.

41. Pierre Damien, Epistolae, éd. K. Reindel, Monumenta Germaniae Historica, Die Briefe der deutschen Kaizerzeit, IV, 3, Munich, 1989, n 110 (1064), p. 239-241.

42. Pierre DAmien, ibid., $\mathrm{n}^{\circ} 170$ (1070), p. 253.

43. Ph. JOBert, La Notion de donation..., p. 159, 163-169.

44. CSV 110 (1014), 113 (1008), CL 154 bis (1033).

45. CSV 123 (1035), 238 (1056), CL 244 (1080-97), 281 (ap. 1109), t. II n 28 (1022), CLU 2731 (v. 1020), 3506 (1077), 3557 (v. 1080), 3585 (1081).

46. CSV 165 (1028), CL 154 (1012), 230 (1022).

47. CLU 2110 (993-1048), 2112 (993-1048), CLU 4070 (v. 1140).

48. J. Wollasch, «Les moines et la mémoire des morts », Religion et culture autour de l'An Mil. Royaume capétien et Lotharingie, D. Iogna-Prat, J.-CH. PiCARD (sous la dir. de), Paris, 1990, p. 47-54 (ici p. 52-53); D. LE BLÉveC, Recherches sur l'assistance dans les pays du Bas-Rhône du XII siècle au milieu du XV' siècle, thèse d'État, université de Paris-IV, 1994.

49. CLU 3404 (1065).

50. M. LaUwers, La mémoire des ancêtres..., p. 179-181; D. Iogna-Prat, Ordonner..., p. $247-248$.

51. F. BougaRD, «Trésors et mobilia italiens... », p. 167, note 40.

52. CSV 175 (1015), 102 (1065-79), CL 81 (1061). 